Article

\title{
Convergence of Generalized Lupaş-Durrmeyer Operators
}

\author{
Mohd Qasim ${ }^{1}\left(\mathbb{D}\right.$, Mohammad Mursaleen ${ }^{2,3,4, *(\mathbb{D})}$, Asif Khan ${ }^{3} \mathbb{D}$ and Zaheer Abbas ${ }^{1} \mathbb{D}$ \\ 1 Department of Mathematical Sciences, Baba Ghulam Shah Badshah University, Rajouri 185234, \\ Jammu and Kashmir, India; bgsbuqasim@gmail.com (M.Q.); az1129200@yahoo.co.in (Z.A.) \\ 2 Department of Medical Research, China Medical University Hospital, China Medical University, \\ Taichung 40402, Taiwan \\ 3 Department of Mathematics, Aligarh Muslim University, Aligarh 202002, India; asifjnu07@gmail.com \\ 4 Department of Computer Science and Information Engineering, Asia University, Taichung 41354, Taiwan \\ * Correspondence: mursaleen.mm@amu.ac.in; Tel.: +91-941-149-1600
}

Received: 9 May 2020; Accepted: 22 May 2020; Published: 24 May 2020

check for updates

\begin{abstract}
The main aim of this paper is to establish summation-integral type generalized Lupaş operators with weights of Beta basis functions which depends on $\mu$ having some properties. Primarily, for these new operators, we calculate moments and central moments, weighted approximation is discussed. Further, Voronovskaya type asymptotic theorem is proved. Finally, quantitative estimates for the local approximation is taken into consideration.
\end{abstract}

Keywords: generalized Lupaş operators; Beta function; Korovkin's type theorem; convergence theorems; Voronovskaya type theorem

\section{Introduction}

In 1995, A. Lupaş [1] introduced a famous linear positive operators as follows:

$$
L_{m}(g ; y)=\sum_{j=1}^{\infty} l_{m, j}(y) g\left(\frac{j}{m}\right), \quad y \geq 0,
$$

where $g$ is defined on $[0, \infty)$. and

$$
l_{m, j}(y)=2^{-m y}\left(\begin{array}{c}
m y+j-1 \\
j
\end{array}\right) 2^{-j}
$$

In order to approximate Lebesgue integrable functions the most important modifications are Kantorovich and Durrmeyer integral operators. The Kantorovich and Durrmeyer varaint of operator (1) is introduced by Agratini [2], the Durremyer variant of operator (1) is defined as follows:

$$
\mathcal{D}_{m}(g ; y)=\sum_{j=1}^{\infty} c_{m, j} l_{m, j}(y) \int_{0}^{\infty} l_{m, j}(t) g(t) d t,
$$

where $l_{m, j}(y)$ is defined in (1) and

$$
c_{m, j}(t)=\frac{1}{\int_{0}^{\infty} l_{m, j}(u) d u} .
$$

Durrmeyer variant of (2) is not easy to handle. Actually, the first and second order derivatives of the Lupaş basis functions come in terms of Stirling numbers because of this it is very tedious to obtain higher order moments. So, keeping this fact in mind various authors define the Durrmeyer variant 
of operator (2) and various other operators have been studied intensively by taking Beta, Szasz or Baskakov basis functions as in [3-8] and the references there in.

In 2011, Cárdenas et al. [9] defined the Bernstein type operators by $B_{m}\left(g \circ \mu^{-1}\right) o \mu$ and also presents a better degree of approximation depending on $\mu$. After, this generalization it becomes interesting to construct such generalization of other operators. In 2014, a similar modification of Szász-Mirakyan type operators is introduced by Aral et al. [10] by using a suitable function $\mu$.

Very recently, a new modification of operators (1) is introduced by İlarslan et al. [11] by using a suitable function $\mu$, which satisfies following properties

$\left(\mu_{1}\right) \quad \mu$ be a continuously differentiable function on $[0, \infty)$,

$\left(\mu_{2}\right) \quad \mu(0)=0$ and $\inf _{y \in[0, \infty)} \mu^{\prime}(y) \geq 1$.

For $g \in[0, \infty)$ the new constructed operators are defined as

$$
\mathcal{L}_{m}^{\mu}(g ; y)=2^{-m \mu(y)} \sum_{j=0}^{\infty} \frac{(m \mu(y))_{j}}{2^{j} j !}\left(g o \mu^{-1}\right)\left(\frac{j}{m}\right)
$$

for $m \geq 1, y \geq 0$. If $\mu(y)=y$ then (3) reduces to operators (1).

Motivated by the above mentoned Durrmeyer type generalizations of Lupaş and some other operators in this paper we introduce Durrmyer type modification of generalized Lupaş operators (3) by taking weights of Beta basis function. Actually we have two type of modifications in our mind which are defined as follows:

$$
\begin{gathered}
\mathcal{D}_{m}^{\mu}(g ; y)=\sum_{j=1}^{\infty} \ell_{m, j}^{\mu}(y) \int_{0}^{\infty} b_{m, j-1}(\xi)\left(g o \mu^{-1}\right)(\xi) d \xi \\
\mathcal{D}^{*}{ }_{m, \mu}(g ; y)=\sum_{j=1}^{\infty} \ell_{m, j}^{\mu}(y) \int_{0}^{\infty} b_{m, j-1}(\xi)\left(g o \mu^{-1}\right)(\xi) d \xi+\ell_{m, 0}^{\mu}\left(g o \mu^{-1}\right)(0)
\end{gathered}
$$

where $g$ is defined on $[0, \infty), m \geq 1, y \geq 0$ and

$$
\ell_{m, j}^{\mu}(y)=2^{-m \mu(y)}\left(\begin{array}{c}
m \mu(y)+j-1 \\
j
\end{array}\right) 2^{-j}, b_{m, j-1}(\xi)=\frac{1}{\beta(j, m+1)} \frac{\xi^{j-1}}{(1+\xi)^{m+j+1}} .
$$

and $\mu$ is a function satisfying the conditions $\left(\mu_{1}\right)$ and $\left(\mu_{2}\right)$ given above.

Opertaor $\mathcal{D}^{*}{ }_{m, \mu}$ is more smoother then $\mathcal{D}_{m}^{\mu}$, the main difference between them is that $\mathcal{D}^{*}{ }_{m, \mu}$ reproduce every linear function while $\mathcal{D}_{m}^{\mu}$ reproduces only constant ones. So, in this paper we will work on operators (5). The present work is organized as follows. In the second section, moments and central moments for $\mathcal{D}^{*}{ }_{m, \mu}$ are calculated. In the third section, we study convergence properties of $\mathcal{D}^{*}{ }_{m, \mu}$ in the light of weighted space. In section fourth, we obtain the order of approximation of new constructed operators associated with the weighted modulus of continuity. In section fifth, we shall show point-wise convergence by proving Voronovskaya type theorem in quantitative form. Finally, in last section, we obtain some local approximation results related to $\mathcal{K}$-functional.

\section{Basic Results}

Here, we shall prove some lemmas for $\mathcal{D}^{*}{ }_{m, \mu}$ which are required to prove our main results. One can prove these lemmas by using this fact

$$
\mathcal{D}^{*}{ }_{m, \mu}\left(\mu^{r}, y\right)=\frac{r !(m-r) ! . m \mu(y)}{m !} \cdot{ }_{2} F_{1}(m \mu(y)+1,1-r ; 2 ;-1),
$$


there are various other methods to prove these lemmas but, we will prove these lemmas by using the elementary hypergeometric functions ${ }_{1} F_{0}(a ;-; x)$ and by using the factorial polynomials, defined as

$$
j^{(n)}=j(j-1)(j-2) \cdots(j-n+1) .
$$

Lemma 1. For $\mathcal{D}^{*}{ }_{m, \mu}$ given by (5). we have

(i) $\mathcal{D}^{*}{ }_{m, \mu}(1 ; y)=1$,

(ii) $\mathcal{D}^{*}{ }_{m, \mu}(\mu ; y)=\mu(y)$,

(iii) $\mathcal{D}^{*}{ }_{m, \mu}\left(\mu^{2} ; y\right)=\frac{m \mu^{2}(y)+3 \mu(y)}{m-1}$,

(iv) $\mathcal{D}^{*}{ }_{m, \mu}\left(\mu^{3} ; y\right)=\frac{m^{2} \mu^{3}(y)+9 m \mu^{2}(y)+14 \mu(y)}{(m-1)(m-2)}$,

(v) $\mathcal{D}^{*}{ }_{m, \mu}\left(\mu^{4} ; y\right)=\frac{m^{3} \mu^{4}(y)+18 m^{2} \mu^{3}(y)+83 m \mu^{2}(y)+90 \mu(y)}{(m-1)(m-2)(m-3)}$.

Proof. By using the identity

$$
\int_{0}^{\infty} b_{m, j-1}(\xi)\left(\xi^{n}\right) d \xi=\int_{0}^{\infty} \frac{1}{\beta(j, m+1)} \frac{\xi^{j-1}}{(1+\xi)^{m+j+1}} d \xi=\frac{(k+m-1) ! .(m-n) !}{m !(j-1) !}
$$

and the fact that

$$
\left.{ }_{1} F_{0}(a ;-; x)=\sum_{j=0}^{\infty}(a)\right)_{j} \frac{x^{j}}{j !}=(1-x)^{-a},|x|<1,
$$

we obtain

(i)

$$
\begin{aligned}
\mathcal{D}^{*}{ }_{m, \mu}(1 ; y) & =\sum_{j=1}^{\infty} \ell_{m, j}^{\mu}(y)+\ell_{m, 0}^{\mu}(y)=\sum_{j=0}^{\infty} \ell_{m, j}^{\mu}(y) \\
& =2^{-m \mu(y)} \sum_{j=0}^{\infty} \frac{(m \mu(y))_{j}}{2^{j} j !}=2^{-m \mu(y)}{ }_{1} F_{0}\left(m \mu(y) ;-; \frac{1}{2}\right) \\
& =2^{-m \mu(y)}\left(1-\frac{1}{2}\right)^{-m \mu(y)}=1 .
\end{aligned}
$$

(ii)

$$
\begin{aligned}
\mathcal{D}^{*}{ }_{m, \mu}(\mu ; y) & =\sum_{j=1}^{\infty} \ell_{m, j}^{\mu}(y) \frac{j}{m}=\frac{2^{-m \mu(y)}}{m} \sum_{j=1}^{\infty} \frac{(m \mu(y))_{j}}{2^{j}(j-1) !} \\
& =\frac{2^{-m \mu(y)}}{m} \sum_{j=0}^{\infty} \frac{(m \mu(y))_{j+1}}{2^{j+1}(j) !}=\frac{2^{-m \mu(y)-1}}{m} \sum_{j=0}^{\infty} \frac{m \mu(y)(m \mu(y)+1)_{j}}{2^{j}(j) !} \\
& =\mu(y) 2^{-m \mu(y)-1}{ }_{1} F_{0}\left(m \mu(y)+1 ;-; \frac{1}{2}\right) \\
& =\mu(y) 2^{-m \mu(y)-1}\left(1-\frac{1}{2}\right)^{-m \mu(y)-1}=\mu(y) .
\end{aligned}
$$


(iii) By writing $j(j+1)$ in terms of factorial polynomials i.e., $j^{2}+j=j^{(2)}+2 j^{(1)}$ and by using rising factorial of $(m \mu(y))_{j+2}=(m \mu(y))(m \mu(y)+1)(m \mu(y)+2)_{j}$ we obtain

$$
\begin{aligned}
\mathcal{D}_{m, \mu}^{*}\left(\mu^{2} ; y\right) & =\sum_{j=1}^{\infty} \ell_{m, j}^{\mu}(y) \frac{j^{2}+j}{m(m-1)}=\sum_{j=1}^{\infty} \ell_{m, j}^{\mu}(y) \frac{j^{(2)}+2 j^{(1)}}{m(m-1)} \\
& =\frac{2^{-m \mu(y)}}{m(m-1)}\left[\sum_{j=2}^{\infty} \frac{(m \mu(y))_{j}}{2^{j}(j-2) !}+\sum_{j=1}^{\infty} \frac{2(m \mu(y))_{j}}{2^{j}(j-1) !}\right] \\
& =\frac{2^{-m \mu(y)}}{m(m-1)}\left[\sum_{j=0}^{\infty} \frac{(m \mu(y))_{j+2}}{2^{j+2}(j) !}+\sum_{j=0}^{\infty} \frac{2(m \mu(y))_{j+1}}{2^{j+1}(j) !}\right] \\
& =\frac{2^{-m \mu(y)}}{m(m-1)}\left[\sum_{j=0}^{\infty} \frac{(m \mu(y))(m \mu(y)+1)(m \mu(y)+2)_{j}}{2^{j+2}(j) !}+\sum_{j=0}^{\infty} \frac{2(m \mu(y))(m \mu(y)+1)_{j}}{2^{j+1}(j) !}\right] \\
& =\frac{2^{-m \mu(y)}}{m(m-1)}\left[\sum_{j=0}^{\infty} \frac{(m \mu(y))(m \mu(y)+1)(m \mu(y)+2)_{j}}{2^{j+2}(j) !}\right] \\
& +\frac{2^{-m \mu(y)}}{m(m-1)}\left[\sum_{j=0}^{\infty} \frac{2(m \mu(y))(m \mu(y)+1)_{j}}{2^{j+1}(j) !}\right] \\
& =\frac{(m \mu(y))(m \mu(y)+1)}{m(m-1)} 2^{-m \mu(y)-2} F_{0}\left(m \mu(y)+2 ;-; \frac{1}{2}\right) \\
& +\frac{2(m \mu(y))}{m(m-1)} 2^{-m \mu(y)-1} F_{0}\left(m \mu(y)+1 ;-; \frac{1}{2}\right) \\
& =\frac{(m \mu(y))(m \mu(y)+1)}{m(m-1)} 2^{-m \mu(y)-2}\left(1-\frac{1}{2}\right) \\
& +\frac{2(m \mu(y))}{m(m-1)} 2^{-m \mu(y)-1}\left(1-\frac{1}{2}\right) \\
& =\frac{m \mu^{2}(y)+3 \mu(y)}{m-1} .
\end{aligned}
$$

(iv)

$$
\begin{aligned}
\mathcal{D}^{*}{ }_{m, \mu}\left(\mu^{3} ; y\right) & =\sum_{j=1}^{\infty} \ell_{m, j}^{\mu}(y) \frac{j^{2}+3 j^{2}+2 j}{m(m-1)(m-2)}=\sum_{j=1}^{\infty} \ell_{m, j}^{\mu}(y) \frac{j^{(3)}+6 j^{(2)}+6 j^{(1)}}{m(m-1)(m-2)} \\
& =\frac{2^{-m \mu(y)}}{m(m-1)(m-2)}\left[\sum_{j=3}^{\infty} \frac{(m \mu(y))_{j}}{2^{j}(j-3) !}+6 \sum_{j=2}^{\infty} \frac{(m \mu(y))_{j}}{2^{j}(j-2) !}+6 \sum_{j=1}^{\infty} \frac{(m \mu(y))_{j}}{2^{j}(j-1) !}\right] \\
& =\frac{2^{-m \mu(y)}}{m(m-1)(m-2)}\left[\sum_{j=0}^{\infty} \frac{(m \mu(y))_{j+3}}{2^{j+3}(j) !}+6 \sum_{j=0}^{\infty} \frac{(m \mu(y))_{j+2}}{2^{j+2}(j) !}+6 \sum_{j=0}^{\infty} \frac{(m \mu(y))_{j+1}}{2^{j+1}(j) !}\right] \\
& =\frac{2^{-m \mu(y)}}{m(m-1)(m-2)}\left[\sum_{j=0}^{\infty} \frac{(m \mu(y))(m \mu(y)+1)(m \mu(y)+2)(m \mu(y)+3)_{j}}{2^{j+3}(j) !}\right] \\
& +\frac{2^{-m \mu(y)}}{m(m-1)(m-2)}\left[6 \sum_{j=0}^{\infty} \frac{(m \mu(y))(m \mu(y)+1)(m \mu(y)+2)_{j}}{2^{j+2}(j) !}\right] \\
& +\frac{2^{-m \mu(y)}}{m(m-1)(m-2)}\left[6 \sum_{j=0}^{\infty} \frac{(m \mu(y))(m \mu(y)+1)_{j}}{2^{j+1}(j) !}\right]
\end{aligned}
$$




$$
\begin{aligned}
& =\frac{(m \mu(y))(m \mu(y)+1)(m \mu(y)+2)}{m(m-1)(m-2)} 2^{-m \mu(y)-3}{ }_{1} F_{0}\left(m \mu(y)+3 ;-; \frac{1}{2}\right) \\
& +\frac{6(m \mu(y))(m \mu(y)+1)}{m(m-1)(m-2)} 2^{-m \mu(y)-2}{ }_{1} F_{0}\left(m \mu(y)+2 ;-; \frac{1}{2}\right) \\
& +\frac{6(m \mu(y))}{m(m-1)(m-2)} 2^{-m \mu(y)-1}{ }_{1} F_{0}\left(m \mu(y)+1 ;-; \frac{1}{2}\right) \\
& =\frac{(m \mu(y))(m \mu(y)+1)(m \mu(y)+2)}{m(m-1)(m-2)} 2^{-m \mu(y)-3}\left(1-\frac{1}{2}\right)^{-m \mu(y)-3} \\
& +\frac{6(m \mu(y))(m \mu(y)+1)}{m(m-1)(m-2)} 2^{-m \mu(y)-2}\left(1-\frac{1}{2}\right)^{-m \mu(y)-2} \\
& +\frac{6(m \mu(y))}{m(m-1)(m-2)} 2^{-m \mu(y)-1}\left(1-\frac{1}{2}\right)^{-m \mu(y)-1} \\
& =\frac{m^{2} \mu^{3}(y)+9 m \mu^{2}(y)+14 \mu(y)}{(m-1)(m-2)} .
\end{aligned}
$$

(v) Finally, by using $j^{4}+6 j^{3}+11 j^{2}+6 j=j^{(4)}+12 j^{(3)}+36 j^{(2)}+18 j^{(1)}$, we have

$$
\begin{aligned}
& \mathcal{D}^{*}{ }_{m, \mu}\left(\mu^{4} ; y\right)=\sum_{j=1}^{\infty} \ell_{m, j}^{\mu}(y) \frac{j^{4}+6 j^{3}+11 j^{2}+6 j}{m(m-1)(m-2)(m-3)}=\sum_{j=1}^{\infty} \ell_{m, j}^{\mu}(y) \frac{j^{(4)}+12 j^{(3)}+36 j^{(2)}+18 j^{(1)}}{m(m-1)(m-2)(m-3)} \\
& =\frac{2^{-m \mu(y)}}{m(m-1)(m-2)(m-3)}\left[\sum_{j=4}^{\infty} \frac{(m \mu(y))_{j}}{2^{j}(j-4) !}+12 \sum_{j=3}^{\infty} \frac{(m \mu(y))_{j}}{2^{j}(j-3) !}+36 \sum_{j=2}^{\infty} \frac{(m \mu(y))_{j}}{2^{j}(j-2) !}\right. \\
& \left.+18 \sum_{j=1}^{\infty} \frac{(m \mu(y))_{j}}{2^{j}(j-1) !}\right] \\
& =\frac{2^{-m \mu(y)}}{m(m-1)(m-2)(m-3)}\left[\sum_{j=4}^{\infty} \frac{(m \mu(y))_{j+4}}{2^{j+4}(j) !}+12 \sum_{j=3}^{\infty} \frac{(m \mu(y))_{j+3}}{2^{j+3}(j) !}\right. \\
& \left.+\quad 36 \sum_{j=2}^{\infty} \frac{(m \mu(y))_{j+2}}{2^{j+2}(j) !}+18 \sum_{j=1}^{\infty} \frac{(m \mu(y))_{j+1}}{2^{j+1}(j) !}\right] \\
& =\frac{2^{-m \mu(y)}}{m(m-1)(m-2)(m-3)} \\
& \times\left[\sum_{j=0}^{\infty} \frac{(m \mu(y))(m \mu(y)+1)(m \mu(y)+2)(m \mu(y)+3)(m \mu(y)+4)_{j}}{2^{j+4}(j) !}\right] \\
& +\frac{2^{-m \mu(y)}}{m(m-1)(m-2)(m-3)}\left[12 \sum_{j=0}^{\infty} \frac{(m \mu(y))(m \mu(y)+1)(m \mu(y)+2)(m \mu(y)+3)_{j}}{2^{j+3}(j) !}\right] \\
& +\frac{2^{-m \mu(y)}}{m(m-1)(m-2)(m-3)}\left[36 \sum_{j=0}^{\infty} \frac{(m \mu(y))(m \mu(y)+1)(m \mu(y)+2)_{j}}{2^{j+2}(j) !}\right] \\
& +\frac{2^{-m \mu(y)}}{m(m-1)(m-2)(m-3)}\left[18 \sum_{j=0}^{\infty} \frac{(m \mu(y))(m \mu(y)+1)_{j}}{2^{j+1}(j) !}\right] \\
& =\frac{(m \mu(y))(m \mu(y)+1)(m \mu(y)+2)(m \mu(y)+3)}{m(m-1)(m-2)(m-3)} 2^{-m \mu(y)-3}{ }_{1} F_{0}\left(m \mu(y)+4 ;-; \frac{1}{2}\right) \\
& +12 \frac{(m \mu(y))(m \mu(y)+1)(m \mu(y)+2)}{m(m-1)(m-2)(m-3)} 2^{-m \mu(y)-4}{ }_{1} F_{0}\left(m \mu(y)+3 ;-; \frac{1}{2}\right) \\
& +\quad 36 \frac{(m \mu(y))(m \mu(y)+1)}{m(m-1)(m-2)(m-3)} 2^{-m \mu(y)-2}{ }_{1} F_{0}\left(m \mu(y)+2 ;-; \frac{1}{2}\right) \\
& +18 \frac{(m \mu(y))}{m(m-1)(m-2)(m-3)} 2^{-m \mu(y)-1}{ }_{1} F_{0}\left(m \mu(y)+1 ;-; \frac{1}{2}\right) \\
& =\frac{(m \mu(y))(m \mu(y)+1)(m \mu(y)+2)(m \mu(y)+3)}{m(m-1)(m-2)(m-3)} 2^{-m \mu(y)-4}\left(1-\frac{1}{2}\right)^{-m \mu(y)-4}
\end{aligned}
$$




$$
\begin{aligned}
& +12 \frac{(m \mu(y))(m \mu(y)+1)(m \mu(y)+2)}{m(m-1)(m-2)(m-3)} 2^{-m \mu(y)-3}\left(1-\frac{1}{2}\right)^{-m \mu(y)-3} \\
& +36 \frac{(m \mu(y))(m \mu(y)+1)}{m(m-1)(m-2)(m-3)} 2^{-m \mu(y)-2}\left(1-\frac{1}{2}\right)^{-m \mu(y)-2} \\
& +\frac{18(m \mu(y))}{m(m-1)(m-2)(m-3)} 2^{-m \mu(y)-1}\left(1-\frac{1}{2}\right)^{-m \mu(y)-1} \\
& =\frac{m^{3} \mu^{4}(y)+18 m^{2} \mu^{3}(y)+83 m \mu^{2}(y)+90 \mu(y)}{(m-1)(m-2)(m-3)} .
\end{aligned}
$$

Hence proved.

Lemma 2. By using linearity of operator $\mathcal{D}^{*}{ }_{m, \mu}$ and by Lemma 1 we have the following central moments

(i) $\mathcal{D}^{*}{ }_{m, \mu}(\mu(\xi)-\mu(y) ; y)=0$,

(ii) $\mathcal{D}^{*}{ }_{m, \mu}\left((\mu(\xi)-\mu(y))^{2} ; y\right)=\frac{\mu^{2}(y)+3 \mu(y)}{m-1}$,

(iii) $\mathcal{D}^{*}{ }_{m, \mu}\left((\mu(\xi)-\mu(y))^{3} ; y\right)=\frac{4 \mu^{3}(y)+18 \mu^{2}(y)+14 \mu(y)}{(m-1)(m-2)}$,

(iv) $\mathcal{D}^{*}{ }_{m, \mu}\left((\mu(\xi)-\mu(y))^{4} ; y\right)=\frac{(3 m+18) \mu^{4}(y)+(18 m+108) \mu^{3}(y)+(27 m+168) \mu^{2}(y)+90 \mu(y)}{(m-1)(m-2)(m-3)}$.

\section{Weighted Estimates}

In this section we prove convergence properties of new constructed operators $\mathcal{D}^{*}{ }_{m, \mu}$ in the light of weighted space.

Let $\Psi(y)$ be a function satisfying the conditions $\left(\mu_{1}\right)$ and $\left(\mu_{2}\right)$ given above. Also, we take the weight function $\Psi(y)=1+\mu^{2}(y)$ and we define the weighted spaces as follows:

$$
\mathcal{B}_{\Psi}[0, \infty)=\left\{g:[0, \infty) \rightarrow \mathbb{R}|| g(y) \mid \leq \mathcal{M}_{g} \Psi(y), y \geq 0\right\}
$$

where $\mathcal{M}_{g}$ is a constant which depends only on $g . \mathcal{B}_{\Psi}[0, \infty)$ is a normed linear space equipped with the norm

$$
\|g\|_{\Psi}=\sup _{y \in[0, \infty)} \frac{|g(y)|}{\Psi(y)} .
$$

Also, the following subspaces of $\mathcal{B}_{\Psi}[0, \infty)$

$$
\begin{gathered}
\mathcal{C}_{\Psi}[0, \infty)=\left\{g \in \mathcal{B}_{\Psi}[0, \infty): \quad g \text { is continuous on }[0, \infty)\right\}, \\
\mathcal{C}_{\Psi}^{*}[0, \infty)=\left\{g \in \mathcal{C}_{\Psi}[0, \infty): \lim _{y \rightarrow \infty} \frac{g(y)}{\Psi(y)}=\mathcal{M}_{g}=\text { Constant }\right\}, \\
U_{\Psi}[0, \infty)=\left\{g \in \mathcal{C}_{\Psi}[0, \infty): \frac{g(y)}{\Psi(y)} \text { is uniformly continuous on }[0, \infty)\right\} .
\end{gathered}
$$

It is Obvious that $\mathcal{C}_{\Psi}^{*}[0, \infty) \subset U_{\Psi}[0, \infty) \subset \mathcal{C}_{\Psi}[0, \infty) \subset \mathcal{B}_{\Psi}[0, \infty)$.

In $[12,13]$, Gadjiev prove the following results for the weighted Korovkin type theorems.

Lemma 3 ([12]). The positive linear operators $\mathcal{Q}_{m}, m \geq 1$, acts from $\mathcal{C}_{\Psi}[0, \infty)$ to $\mathcal{B}_{\Psi}[0, \infty)$ if and only if the inequality

$$
\left|\mathcal{Q}_{m}(\Psi ; y)\right| \leq \mathcal{M}_{m} \Psi(y), y \geq 0,
$$

holds, where $\mathcal{M}_{m}>0$ is a positive constant.

Theorem 1 ([13]). Let the sequence of positive linear operators $\mathcal{Q}_{m}, m \geq 1$ acting from $\mathcal{C}_{\Psi}[0, \infty)$ to $\mathcal{B}_{\Psi}[0, \infty)$ and satisfying

$$
\lim _{m \rightarrow \infty}\left\|\mathcal{Q}_{m} \mu^{r}-\mu^{r}\right\|_{\Psi}=0, \quad r=0,1,2 .
$$


Then for each $g \in C_{\Psi}^{*}[0, \infty)$ we have

$$
\lim _{m \rightarrow \infty}\left\|\mathcal{Q}_{m}(g)-g\right\|_{\Psi}=0 .
$$

Theorem 2. For each function $g \in C_{\Psi}^{*}[0, \infty)$ we have

$$
\lim _{m \rightarrow \infty}\left\|\mathcal{D}^{*}{ }_{m, \mu}(g)-g\right\|_{\Psi}=0 .
$$

Proof. From Lemma 1, we have

$$
\left\|\mathcal{D}^{*}{ }_{m, \mu}(1 ; y)-1\right\|_{\Psi}=0,
$$

and,

$$
\left\|\mathcal{D}^{*}{ }_{m, \mu}(\mu ; y)-\mu\right\|_{\Psi}=0 .
$$

Also,

$$
\begin{aligned}
\left\|\mathcal{D}^{*}{ }_{m, \mu}\left(\mu^{2} ; y\right)-\mu^{2}\right\|_{\Psi} & =\sup _{y \in[0, \infty)}\left(\frac{\mu^{2}(y)+3 \mu(y)}{(m-1)\left(1+\mu^{2}(y)\right)}\right) \\
& \leq \frac{4}{m-1}
\end{aligned}
$$

We deduce

$$
\lim _{m \rightarrow \infty}\left\|\mathcal{D}^{*}{ }_{m, \mu}(g)-g\right\|_{\Psi}=0 .
$$

by Theorem 1 .

\section{Rate of Convergence}

In this part, we would like to determine the rate of convergence for $\mathcal{D}^{*}{ }_{m, \mu}$ by weighted modulus of continuity $\omega_{\mu}(f ; \lambda)$ which was introduced by Holhos [14] in 2008, as follows:

$$
\omega_{\mu}(g ; \lambda)=\sup _{y, \xi \in[0, \infty),|\mu(\xi)-\mu(y)| \leq \lambda} \frac{|g(\xi)-g(y)|}{\Psi(\xi)+\Psi(y)}, \quad \lambda>0,
$$

where $g \in \mathcal{C}_{\Psi}[0, \infty)$, having following properties:

(i) $\omega_{\mu}(g ; 0)=0$,

(ii) $\omega_{\mu}(g ; \lambda) \geq 0, \lambda \geq 0$ for $g \in \mathcal{C}_{\Psi}[0, \infty)$,

(iii) $\lim _{\lambda \rightarrow 0} \omega_{\mu}(g ; \lambda)=0$, for each $g \in U_{\Psi}[0, \infty)$.

Theorem 3 ([14]). Let $\mathcal{Q}_{m}: \mathcal{C}_{\Psi}[0, \infty) \rightarrow \mathcal{B}_{\Psi}[0, \infty)$ be a sequence of positive linear operators with

$$
\begin{aligned}
\left\|\mathcal{Q}_{m}\left(\mu^{0}\right)-\mu^{0}\right\|_{\Psi^{0}} & =a_{m}, \\
\left\|\mathcal{Q}_{m}(\mu)-\mu\right\|_{\Psi^{\frac{1}{2}}} & =b_{m}, \\
\left\|\mathcal{Q}_{m}\left(\mu^{2}\right)-\mu^{2}\right\|_{\Psi} & =c_{m}, \\
\left\|\mathcal{Q}_{m}\left(\mu^{3}\right)-\mu^{3}\right\|_{\Psi^{\frac{3}{2}}} & =d_{m},
\end{aligned}
$$


where the sequences $a_{m}, b_{m}, c_{m}$ and $d_{m}$ converge to zero as $m \rightarrow \infty$. Then

$$
\left\|\mathcal{Q}_{m}(g)-g\right\|_{\Psi^{\frac{3}{2}}} \leq\left(7+4 a_{m}+2 c_{m}\right) \omega_{\mu}\left(g ; \lambda_{m}\right)+\|g\| \Psi a_{m},
$$

for all $g \in \mathcal{C}_{\Psi}[0, \infty)$, where

$$
\lambda_{m}=2 \sqrt{\left(a_{m}+2 b_{m}+c_{m}\right)\left(1+a_{m}\right)}+a_{m}+3 b_{m}+3 c_{m}+d_{m} .
$$

Theorem 4. For all $g \in \mathcal{C}_{\Psi}[0, \infty)$ we have

$$
\left\|\mathcal{D}^{*}{ }_{m, \mu}(g)-g\right\|_{\Psi^{\frac{3}{2}}} \leq\left(7+\frac{8}{m-1}\right) \omega_{\mu}\left(g ; \lambda_{m}\right),
$$

where

$$
\lambda_{m}=2 \sqrt{\frac{4}{m-1}}+\frac{12}{m-1}+\frac{12 m+14}{(m-1)(m-2)} .
$$

Proof. We should calculate the sequences $\left(a_{m}\right),\left(b_{m}\right),\left(c_{m}\right)$ and $\left(d_{m}\right)$, in order to apply Theorem 3 . In light of Lemma 1 clearly we have

$$
\begin{gathered}
a_{m}=\left\|\mathcal{D}^{*}{ }_{m, \mu}\left(\mu^{0}\right)-\mu^{0}\right\|_{\Psi^{0}}=0, \\
b_{m}=\left\|\mathcal{D}^{*}{ }_{m, \mu}(\mu)-\mu\right\|_{\Psi^{\frac{1}{2}}}=0 .
\end{gathered}
$$

Also,

$$
c_{m}=\left\|\mathcal{D}_{m, \mu}^{*}\left(\mu^{2}\right)-\mu^{2}\right\|_{\Psi} \leq \frac{4}{m-1} .
$$

Finally,

$$
d_{m}=\left\|\mathcal{D}^{*}{ }_{m, \mu}\left(\mu^{3}\right)-\mu^{3}\right\|_{\Psi^{\frac{3}{2}}} \leq \frac{12 m+14}{(m-1)(m-2)} .
$$

Thus all the conditions Theorem 3 are satisfied, the desired result follows.

Remark 1. For $\lim _{\lambda \rightarrow 0} \omega_{\mu}(g ; \lambda)=0$ in Theorem 4 , we get

$$
\lim _{m \rightarrow \infty}\left\|\mathcal{D}^{*}{ }_{m, \mu}(g)-g\right\|_{\Psi^{\frac{3}{2}}}=0, \text { for } g \in U_{\Psi}[0, \infty) .
$$

\section{Pontwise Convergence $\mathcal{D}^{*}{ }_{m, \mu}$}

In this section, we shall analyze pointwise convergence of $\mathcal{D}^{*}{ }_{m, \mu}$ by obtaining the Voronovskaya theorem in quantitative form by using a same technique in [9].

Theorem 5. Let $g \in \mathcal{C}_{\Psi}[0, \infty), \quad y \in[0, \infty)$ and suppose that $\left(g \circ \mu^{-1}\right)^{\prime}$ and $\left(g o \mu^{-1}\right)^{\prime \prime}$ exist at $\mu(y)$. If $\left(g \circ \mu^{-1}\right)^{\prime \prime}$ is bounded on $[0, \infty)$, then we have

$$
\lim _{m \rightarrow \infty} m\left[\mathcal{D}^{*}{ }_{m, \mu}(g ; y)-g(y)\right]=\frac{1}{2} \mu^{2}(y)\left(g o \mu^{-1}\right)^{\prime \prime} \mu(y)+\frac{3}{2} \mu(y)\left(g \circ \mu^{-1}\right)^{\prime \prime} \mu(y) .
$$


Proof. By Taylor expansion, we have

$$
\begin{aligned}
g(\xi)=\left(g \circ \mu^{-1}\right)(\mu(\xi)) & =\left(g \circ \mu^{-1}\right)(\mu(y))+\left(g \circ \mu^{-1}\right)^{\prime}(\mu(y))(\mu(\xi)-\mu(y)) \\
& +\frac{\left(g \circ \mu^{-1}\right)^{\prime \prime}(\mu(y))(\mu(\xi)-\mu(y))^{2}}{2}+\chi_{y}(\xi)(\mu(\xi)-\mu(y))^{2},
\end{aligned}
$$

where

$$
\chi_{y}(\xi)=\frac{\left(g o \mu^{-1}\right)^{\prime \prime}(\mu(\xi))-\left(g o \mu^{-1}\right)^{\prime \prime}(\mu(y))}{2} .
$$

Therefore, by (15) together with the assumption on $g$ ensures that

$$
\left|\chi_{y}(\xi)\right| \leq \mathcal{K}, \text { for all } \xi \in[0, \infty)
$$

and is convergent to zero as $\xi \rightarrow y$. Now by applying the operators (5) to the equality (14), we get

$$
\begin{aligned}
{\left[\mathcal{D}^{*}{ }_{m, \mu}(g ; y)-g(y)\right] } & =\left(g \circ \mu^{-1}\right)^{\prime}(\mu(y)) \mathcal{D}^{*}{ }_{m, \mu}((\mu(\xi)-\mu(y)) ; y) \\
& +\frac{\left(g o \mu^{-1}\right)^{\prime \prime}(\mu(y)) \mathcal{D}^{*}{ }_{m, \mu}\left((\mu(\xi)-\mu(y))^{2} ; y\right)}{2} \\
& +\mathcal{D}^{*}{ }_{m, \mu}\left(\chi^{y}(\xi)\left((\mu(\xi)-\mu(y))^{2} ; y\right)\right) .
\end{aligned}
$$

From Lemma 2, we obtain

$$
\lim _{m \rightarrow \infty} m \mathcal{D}^{*}{ }_{m, \mu}((\mu(\xi)-\mu(y)) ; y)=0,
$$

also,

$$
\lim _{m \rightarrow \infty} m \mathcal{D}^{*}{ }_{m, \mu}\left((\mu(\xi)-\mu(y))^{2} ; y\right) \leq \mu^{2}(y)+3 \mu(y) .
$$

we will get the proof of the theorem by estimating the last term on the right hand side of equality (16). From (15), for every $\epsilon>0, \lim _{\xi \rightarrow y} \chi_{y}(\xi)=0$. Let $\delta>0$ such that $\left|\chi_{y}(\xi)\right|<\epsilon$ for every $\xi \geq 0$. By Cauchy-Schwartz inequality, we get

$$
\begin{aligned}
\lim _{m \rightarrow \infty} m \mathcal{D}^{*}{ }_{m, \mu}\left(\left|\chi_{y}(\xi)\right|(\mu(\xi)-\mu(y))^{2} ; y\right) & \leq \epsilon \lim _{m \rightarrow \infty} m \mathcal{D}^{*}{ }_{m, \mu}\left((\mu(\xi)-\mu(y))^{2} ; y\right) \\
& +\frac{\mathcal{K}}{\delta^{2}} \lim _{m \rightarrow \infty} \mathcal{D}^{*}{ }_{m, \mu}\left((\mu(\xi)-\mu(y))^{4} ; y\right)
\end{aligned}
$$

Since

$$
\lim _{m \rightarrow \infty} m \mathcal{D}^{*}{ }_{m, \mu}\left((\mu(\xi)-\mu(y))^{4} ; y\right)=0,
$$

we obtain

$$
\lim _{m \rightarrow \infty} m \mathcal{D}^{*}{ }_{m, \mu}\left(\left|\chi_{y}(\xi)\right|(\mu(\xi)-\mu(y))^{2} ; y\right)=0 .
$$

Thus, by taking into account the Equations (17), (18) and (20) to Equation (16) which completes the proof. 


\section{Local Approximation}

In this section, for operator $\mathcal{D}^{*}{ }_{m, \mu}$ we shall present local approximation theorems. Let $\mathcal{C}_{B}[0, \infty)$, denote the space of real-valued continuous and bounded functions $g$ defined on the interval $[0, \infty)$. The norm $\|\cdot\|$ on the space $\mathcal{C}_{B}[0, \infty)$ is defined by

$$
\|g\|=\sup _{0 \leq y<\infty}|g(y)| .
$$

$\mathcal{K}$-functional is defined as:

$$
\mathcal{K}_{2}(g, \lambda)=\inf _{p \in W^{2}}\left\{\|g-p\|+\lambda\left\|g^{\prime \prime}\right\|\right\},
$$

where $\lambda>0$ and $W^{2}=\left\{p \in \mathcal{C}_{B}[0, \infty): p^{\prime}, p^{\prime \prime} \in \mathcal{C}_{B}[0, \infty)\right\}$. By Devore and Lorentz ([15], p. 177, Theorem 2.4), there exists an absolute constant $\mathcal{C}>0$ such that

$$
\mathcal{K}(g, \lambda) \leq \mathcal{C} \omega_{2}(g, \sqrt{\lambda})
$$

also, modulus of smoothness of Second order is given as

$$
\omega_{2}(g, \sqrt{\lambda})=\sup _{0<h \leq \sqrt{\lambda}} \sup _{y \in[0, \infty)}|g(y+2 h)-2 g(y+h)+g(y)|
$$

where $g \in C_{B}[0, \infty)$. For $g \in C_{B}[0, \infty)$ the usual modulus of continuity is defined as

$$
\omega(g, \lambda)=\sup _{0<h \leq \lambda} \sup _{y \in[0, \infty)}|g(y+h)-g(y)| .
$$

Theorem 6. Let $g \in \mathcal{C}_{B}[0, \infty)$. Let $\mu$ be a function satisfying the conditions $\left(\mu_{1}\right),\left(\mu_{2}\right)$ and $\left\|\mu^{\prime \prime}\right\|$ is finite. Then, there exists an absolute constant $\mathcal{C}>0$ such that

$$
\left|\mathcal{D}^{*}{ }_{m, \mu}(g ; y)-g(y)\right| \leq \mathcal{C K}\left(g, \frac{\mu^{2}(y)+3 \mu(y)}{m-1}\right)
$$

Proof. Let $p \in W^{2}$ and $y, \xi \in[0, \infty)$. By Taylor's formula we obtain

$$
p(\xi)=p(y)+\left(p o \mu^{-1}\right)^{\prime}(\mu(y))(\mu(\xi)-\mu(y))+\int_{\mu(y)}^{\mu(\xi)}(\mu(\xi)-v)\left(p o \mu^{-1}\right)^{\prime \prime}(v) d v
$$

By using the equality

$$
\left(p o \mu^{-1}\right)^{\prime \prime}(\mu(y))=\frac{p^{\prime \prime}(y)}{\left(\mu^{\prime}(y)\right)^{2}}-p^{\prime \prime}(y) \frac{\mu^{\prime \prime}(y)}{\left(\mu^{\prime}(y)\right)^{3}} .
$$

Now, put $v=\mu(y)$ in the last term in equality (22), we get

$$
\begin{aligned}
\int_{\mu(y)}^{\mu(\xi)}(\mu(\xi)-v)\left(p o \mu^{-1}\right)^{\prime \prime}(v) d v & =\int_{y}^{\xi}(\mu(\xi)-\mu(y))\left[\frac{p^{\prime \prime}(y) \mu^{\prime}(y)-p^{\prime}(y) \mu^{\prime \prime}(v)}{\left(\mu^{\prime}(y)\right)^{2}}\right] d y \\
& =\int_{\mu(y)}^{\mu(\xi)}(\mu(\xi)-v) \frac{p^{\prime \prime}\left(\mu^{-1}(v)\right)}{\left(\mu^{\prime}\left(\mu^{-1}(v)\right)^{2}\right.} d v \\
& -\int_{\mu(y)}^{\mu(\xi)}(\mu(\xi)-v) \frac{p^{\prime}\left(\mu^{-1}(v)\right) \mu^{\prime \prime}\left(\mu^{-1}(v)\right)}{\left(\mu^{\prime}\left(\mu^{-1}(v)\right)^{3}\right.} d v .
\end{aligned}
$$


By applying operator (5) to the both sides of equality (22), and from Lemma 1 we deduce

$$
\begin{aligned}
\mathcal{D}^{*}{ }_{m, \mu}(p ; y) & =p(y)+\mathcal{D}^{*}{ }_{m, \mu}\left(\int_{\mu(y)}^{\mu(\xi)}(\mu(\xi)-v) \frac{p^{\prime \prime}\left(\mu^{-1}(v)\right)}{\left(\mu^{\prime}\left(\mu^{-1}(v)\right)^{2}\right.} d v ; y\right) \\
& -\mathcal{D}^{*}{ }_{m, \mu}\left(\int_{\mu(y)}^{\mu(\xi)}(\mu(\xi)-v) \frac{p^{\prime}\left(\mu^{-1}(v)\right) \mu^{\prime \prime}\left(\mu^{-1}(v)\right)}{\left(\mu^{\prime}\left(\mu^{-1}(v)\right)^{3}\right.} d v ; y\right) .
\end{aligned}
$$

As we know $\mu$ is strictly increasing on $[0, \infty)$ and with condition $\left(\mu_{2}\right)$, we get

$$
\left|\mathcal{D}^{*}{ }_{m, \mu}(p ; y)-p(y)\right| \leq \mathcal{M}_{m, 2}^{\mu}(y)\left(\left\|p^{\prime \prime}\right\|+\left\|p^{\prime}\right\|\left\|\mu^{\prime \prime}\right\|\right),
$$

where

$$
\mathcal{M}_{m, 2}^{\mu}(y)=\mathcal{D}^{*}{ }_{m, \mu}\left((\mu(\xi)-\mu(y))^{2} ; y\right)
$$

Also, it is clear that

$$
\left|\mathcal{D}^{*}{ }_{m, \mu}\right| \leq\|g\|
$$

Hence we have

$$
\begin{aligned}
\left|\mathcal{D}^{*}{ }_{m, \mu}(g ; y)-g(y)\right| & \leq\left|\mathcal{D}^{*}{ }_{m, \mu}(g-p ; y)\right|+\left|\mathcal{D}^{*}{ }_{m, \mu}(p ; y)-p(y)\right|+|p(y)-g(y)| \\
& \leq 2\|g-p\|+\left\{\frac{\mu^{2}(y)+3 \mu(y)}{m-1}\right\}\left(\left\|p^{\prime \prime}\right\|+\left\|p^{\prime}\right\|\left\|\mu^{\prime \prime}\right\|\right),
\end{aligned}
$$

if $\mathcal{C}=\max \left\{2,\left\|\mu^{\prime \prime}\right\|\right\}$, then

$$
\left|\mathcal{D}^{*}{ }_{m, \mu}(g ; y)-g(y)\right| \leq \mathcal{C}\left(2\|g-p\|+\left\{\frac{\mu^{2}(y)+3 \mu(y)}{m-1}\right\}\left\|p^{\prime \prime}\right\|_{W^{2}}\right) .
$$

Taking infimum over all $p \in W^{2}$ we obtain

$$
\left|\mathcal{D}^{*}{ }_{m, \mu}(g ; y)-g(y)\right| \leq \mathcal{C K}\left(g, \frac{\mu^{2}(y)+3 \mu(y)}{m-1}\right) .
$$

\section{Conclusions}

Here, to approximate Lebesgue integrable function, Durrmeyer variant of the generalized Lupas operators are constructed. We have investigated convergence properties, order of approximation, Voronovskaja type results and also quantitative estimates for the local approximation. The constructed operators have better flexibility and rate of convergence which are depending on the selection of the function $\mu$. Also, the basis of these operators can be used to draw curves and surfaces in Computer Aided Geometric Design (CAGD) [16-18].

Author Contributions: Supervision, A.K. and Z.A.;Writing-original draft, M.Q.; Writing-review and editing, M.M. All authors have read and agreed to the published version of the manuscript.

Funding: This research received no external funding.

Acknowledgments: The first author is grateful to Council of Scientific and Industrial Research (CSIR), India, for providing the Senior Research Fellowship with file no. (09/1172(0001)/2017-EMR-I).

Conflicts of Interest: We declare that there is no conflict of interest. 


\section{References}

1. Lupaş, A. The approximation by some positive linear operators. In Proceedings of the International Dortmund Meeting on Approximation Theory, Witten, Germany, 13-17 March 1995; pp. 201-229.

2. Agratini, O. On the rate of convergence of a positive approximation process. Nihonkai Math. J. 2000, 11, 47-56.

3. Acar, T.; Aral, A.; Rasa, I. Modified Bernstein-Durrmeyer operators. Gen. Math. 2014, 22, 27-41

4. Acar, T.; Mohiudine, S.A.; Mursaleen, M. Approximation by $(p, q)$-Baskakov Durrmeyer Stancu operators. Complex Anal. Oper. Theory 2018, 12, 1453-1468. [CrossRef]

5. Ansari, K.J.; Mursaleen, M.; Rahman, S. Approximation by Jakimovski-Leviatan operators of Durrmeyer type involving multiple Appell polynomials. Rev. Real Acad. Cienc. Exactas Fis. Nat. Ser. A Mat. RACSAM 2019, 113, 1007-1024. [CrossRef]

6. Gupta, V.; Yadav, R. On approximation of certain integral operators. Acta Math. Vietnam. 2014, 39, $193-203$. [CrossRef]

7. Mursaleen, M.; Rahman, S.; Ansari, K.J. Approximation by Jakimovski-Leviatan-Stancu-Durrmeyer type operators. Filomat 2019, 33, 1517-1530. [CrossRef]

8. Mursaleen, M.; Khan, T. On approximation by Stancu type Jakimovski-Leviatan-Durrmeyer operators. Azerbaijan J. Math. 2017, 7 , 16-26. [CrossRef]

9. Cárdenas,M.D.; Garrancho, P.; Rasa, I. Bernstein-type operators which preserve polynomials. Comput. Math. Appl. 2011, 62, 158-163.

10. Aral, A.; Inoan, D.; Rasa, I. On the generalized Szász-Mirakyan operators. Results Math. 2008, 65, 441-452. [CrossRef]

11. İlarslan, H.G.I.; Ali, A.; Gülen, B. Generalized Lupaş operators. AIP Conf. Proc. 2018, 1926, 020019.

12. Gadzhiev, A.D. A problem on the convergence of a sequence of positive linear operators on unbounded sets, and theorems that are analogous to P. P. Korovkin's theorem. Dokl. Akad. Nauk SSSR 1974, 218, 1001-1004. (In Russian)

13. Gadjiev, A.D. Theorems of the type of P. P. Korovkin's theorems. Math. Zamet 1976, 20, 781-786.

14. Holhoş, A. Quantitative estimates for positive linear operators in weighted spaces. Gen. Math. 2008, 16, 99-110.

15. DeVore, R.A.; Lorentz, G.G. Constructive Approximation, Grundlehren Math. Wiss. [Fundamental Principales of Mathematical Sciences]; Springer: Berlin, Germany, 1993.

16. Khan, K.; Lobiyal, D.K. Bézier curves based on Lupaş $(p, q)$-analogue of Bernstein functions in CAGD. J. Comput. Appl. Math. 2017, 317, 458-477. [CrossRef]

17. Khan, K.; Lobiyal, D.K.; Kilicman, A. A de Casteljau Algorithm for Bernstein type Polynomials based on $(p, q)$-integers. Appl. Appl. Math. 2018, 13, 997-1017.

18. Khan, K.; Lobiyal, D.K.; Kilicman, A. Bézier curves and surfaces based on modified Bernstein polynomials. Azerbaijan J. Math. 2019, 9, 3-21.

(C) 2020 by the authors. Licensee MDPI, Basel, Switzerland. This article is an open access article distributed under the terms and conditions of the Creative Commons Attribution (CC BY) license (http://creativecommons.org/licenses/by/4.0/). 